\title{
Laser experiments to simulate supernova remnants*
}

\author{
R. P. Drake, ${ }^{\dagger, \text { a) }}$ J. J. Carroll III, T. B. Smith, and P. Keiter \\ University of Michigan, Ann Arbor, Michigan 48109-2143 \\ S. Gail Glendinning, Omar Hurricane, Kent Estabrook, D. D. Ryutov, B. A. Remington, \\ and R. J. Wallace \\ Lawrence Livermore National Laboratory, P.O. Box 808, Livermore, California 94551 \\ Eli Michael and R. McCray \\ JILA, University of Colorado, Boulder, Colorado 80309
}

(Received 17 November 1999; accepted 17 January 2000)

\begin{abstract}
An experiment using a large laser facility to simulate young supernova remnants (SNRs) is discussed. By analogy to the SNR, the laboratory system includes dense matter that explodes, expansion and cooling to produce energetic, flowing plasma, and the production of shock waves in lower-density surrounding matter. The scaling to SNRs in general and to SN1987A in particular is reviewed. The methods and results of X-ray radiography, by which the system in diagnosed, are discussed. The data show that the hohlraum used to provide the energy for explosion does so in two ways - first, through its radiation pulse, and second, through an additional impulse that is attributed to stagnation pressure. Attempts to model these dynamics are discussed. (C) 2000 American Institute of Physics. [S1070-664X(00)93105-7]
\end{abstract}

\section{INTRODUCTION}

We have recently begun using large lasers, developed for the laser fusion program, ${ }^{1-3}$ to simulate astrophysical phenomena including supernova remnants. The essential feature of such lasers, that makes this possible, is their ability to focus unprecedented amounts of energy into sub-mm volumes. This, in turn, can produce and compress ionized matter under conditions that scale well to astrophysical applications. Our goal in such research is threefold. First, to the extent we can obtain rigorous and complete scaling, we can observe in the laboratory the same phenomena that are present in the stars. Second, to the extent that we can come close to rigorously scaled conditions, we can produce results suitable to test the computational simulation codes used to interpret astrophysical data. There is a need for such tests. Such codes often have not been tested by comparison with hard data, and different codes do not give identical results. Third, in the course of this work we often produce systems that are of fundamental interest, probing new areas of compressible nonlinear hydrodynamics. Our work to simulate young supernova remnants is the subject of the present paper.

Lasers have been applied to the simulation of supernovae, supernova remnants, and related systems for a number of years. There is little point in trying to make complete "model supernovae" in the laboratory, as the global physical system cannot be meaningfully scaled. As reviewed by Drake, ${ }^{4}$ the more recent experiments have tended to choose a physical issue and to attempt to preform a meaningful laboratory simulation that addresses that issue. This has included,

*Paper QI13 Bull. Am. Phys. Soc. 44, 223 (1999).

${ }^{\dagger}$ Invited speaker.

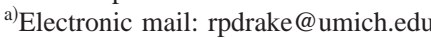

for example, blast-wave instability experiments, ${ }^{5}$ explosive interface instability experiments, ${ }^{6,7}$ and fuel burning experiments. ${ }^{8,9}$ Remington and coauthors have reviewed the broad scope of such work. ${ }^{10,11}$

In the following, we first discuss our approach to the simulation of supernova remnants, and to the comparison with the specific case of SN1987A, which has motivated these studies. Then, we discuss the experimental methods and present the experimental results. Next, we discuss these results and the analysis of them, after which we conclude. This paper builds upon two prior publications, the first of which discussed the design of the experiments ${ }^{12}$ and the second of which ${ }^{13}$ presented data regarding the shock waves driven in the system. Here, we address some differences between the data and the simulations, reported previously, in two ways. We present new evidence regarding the behavior of the mass source for the system, and we present further evidence regarding the equation of state of the foam used as "low-density" matter.

\section{APPROACH TO SUPERNOVA REMNANT SIMULATION}

A supernova remnant (SNR) is an example of a physical system driven by supersonic, flowing plasma. In the formation of an SNR, energetic supernova (SN) ejecta expand into the circumstellar matter, sweeping it up. ${ }^{14,15}$ The resulting strong shock hydrodynamics is responsible for many of the dramatic objects we now observe. As the SN ejecta expand into and are decelerated by the surrounding medium, Rayleigh-Taylor (RT)-induced spike formation leads to clumping. ${ }^{16}$ It may lead eventually to the formation of knots of matter, like those observed in Cassiopeia $\mathrm{A},{ }^{17}$ or to "hydrodynamic bullets" such as those observed in the Orion molecular cloud. ${ }^{18-20}$ 


\section{SN $1987 A$ in 1997}

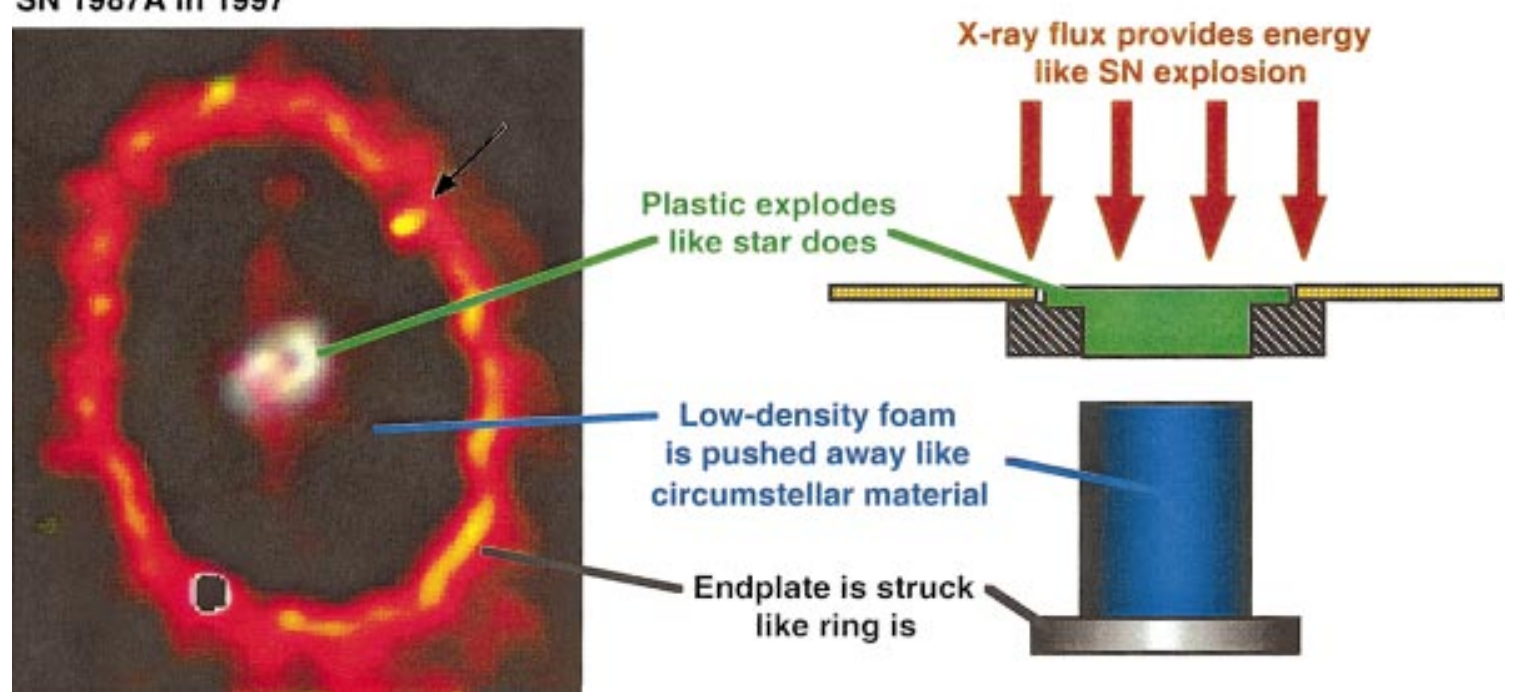

FIG. 1. (Color) A schematic of the experiment and of its relation to SN1987A. The arrow on the image of SN1987A shows the hot spot where interaction of the shocked matter and the ring has begun. In the experiment, the $\mathrm{x}$ rays are produced by a laser-irradiated hohlraum. The image of SN1987A is from the Hubble Space Telescope. It was created with support to the Space Telescope Science Institute, operated by the Association of Universities for Research in Astronomy, Inc., from NASA Contract No. NAS5-26555, and is reproduced with permission from AURA/STScI.

An example of particular current interest is the very young SNR now developing from SN1987A. A recent image of this object is shown on the left in Fig. 1. There is no widely accepted model of the circumstellar ring, which might have arisen from a protostellar disk, ${ }^{21}$ from interacting stellar winds, ${ }^{22-24}$ or from other causes. At present, the ejecta from SN1987A, a high-Mach-number plasma flow, are plowing the residual stellar wind toward the ring. For some time, there has been evidence of interaction with the denser matter near the ring, ${ }^{25,26}$ and recently the first collision of the driven shocks with the ring, at one location, has been observed. ${ }^{27}$ Astrophysicists plan to use the impending collision as a probe of what the ring is. ${ }^{28-30}$ To succeed at this, it is essential to understand the hydrodynamic assembly formed by the ejecta-wind interaction. Recent modeling has shown that this structure will greatly affect the X-ray emission produced in the collision. ${ }^{29}$ Our experiments and the related analysis are intended to improve the interpretation of these data.

Figure 1 shows a schematic of the experiment, and its qualitative relation to SN1987A. The experimental system has much in common with the astrophysical one. In the supernova a blast wave, driven by the explosive release of energy, launches ejecta outward from the star. In the experiments, a strong shock, driven by x-ray ablation, launches ejecta outward from a layer of plastic into vacuum. In both cases: (1) the ejecta expand and cool to produce a lowpressure but high-Mach-number plasma flow; (2) the ejecta drives a strong forward shock into the nearby matter; (3) a reverse shock forms where these ejecta stagnate against the moving interface with this matter; (4) the interface between the ejecta and this matter is unstable to the Rayleigh-Taylor (RT) instability.

Our analysis of the scaling from the SNR to the laboratory has been discussed in a recent article. ${ }^{31} \mathrm{We}$ summarize this discussion here and provide one new, relevant comparison. Both the SNR and the laboratory experiment can be modeled by the Euler equations. This is valid because in both cases: (1) the plasma is well localized, by collisions in the lab and magnetization in the SNR; (2) viscosity is small (high Reynolds number); (3) electron heat conduction is small (high Peclet number); (4) radiation cooling is small. Under these conditions, two systems behave identically if their density, $\rho$, pressure, $p$, and velocity, $u$, profiles have the same normalized shape, and if the ratio $u / \sqrt{p / \rho}$, with the parameters chosen at appropriate corresponding locations in the two systems, is the same. This ratio, the "Euler number,' ' is discussed more thoroughly ${ }^{31}$ in Ryutov et al. It can often be equal to a downstream Mach number. The Euler number is the same in SN1987A and in our experiment; the profiles are similar, although not identical. Figure 2 shows these profiles, comparing hydrocode simulations of the experiments, from simulations discussed previously, ${ }^{13}$ with profiles for the SNR calculated as described in Chevalier, ${ }^{14}$ for parameters appropriate to SN1987A during the interaction with the ionized $\mathrm{H}$ near the ring. ${ }^{25}$ Finally, our planar experiment can represent only a small segment of the spherically expanding SNR, and only on time scales over which
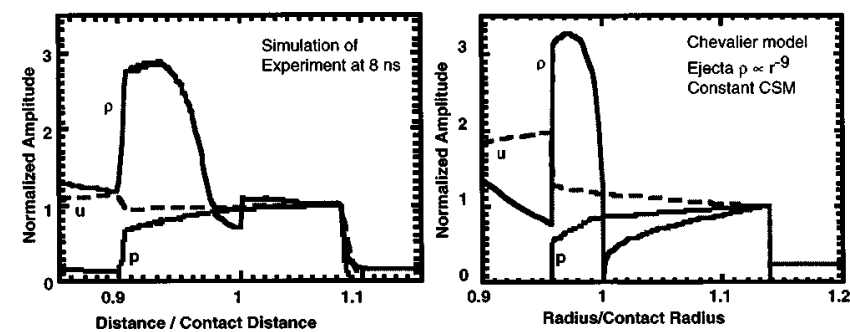

FIG. 2. A comparison of the spatial profiles in the experiment and in a standard model of SN1987A. Profiles of density $(\rho)$, velocity $(u)$, and pressure $(p)$ are shown from a simulation of the experiment (left) and from a standard model of an SNR (right). Here, CSM refers to circumstellar matter and $r$ to radius. 
(a)

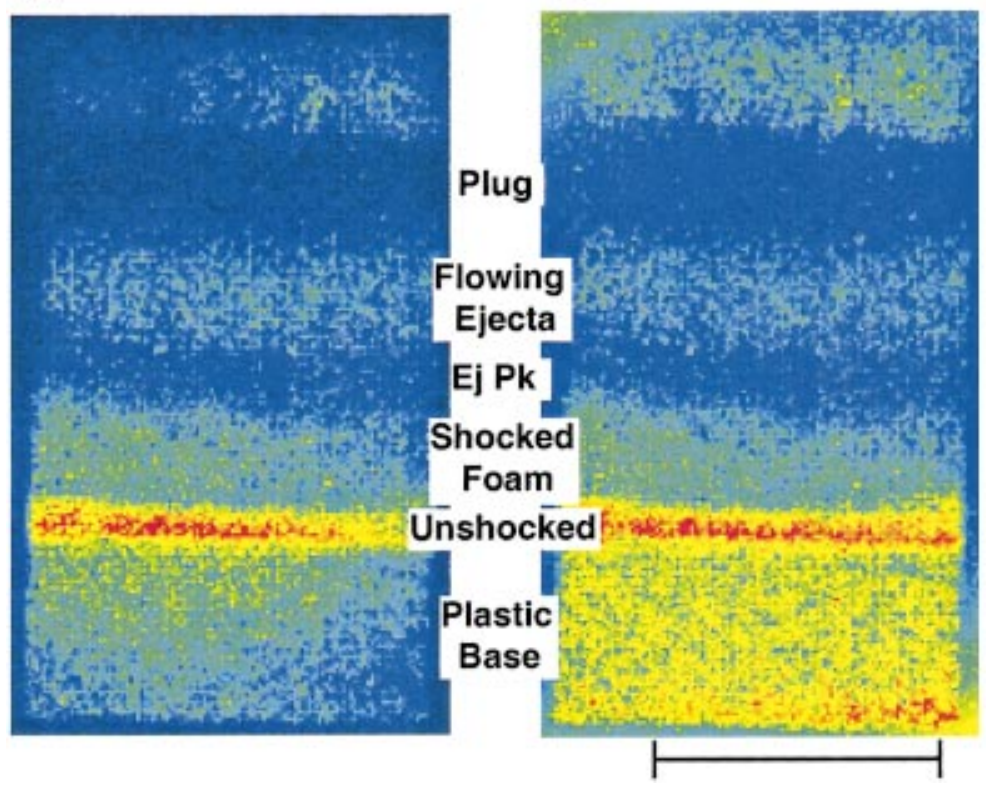

(c)

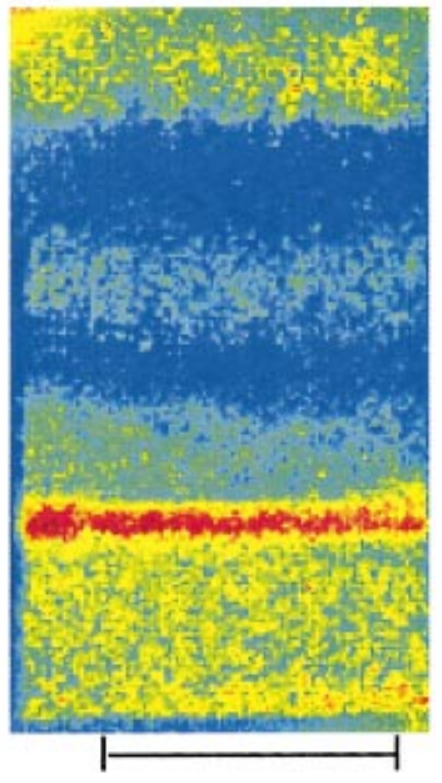

FIG. 3. (Color) Raw (a) and reduced (b), (c) radiography data at $9.11 \mathrm{~ns}$. The features in the target structure are labeled, with "Ej Pk' representing " ejecta peak" and "unshocked" representing "unshocked foam." The data reduction is discussed in the text. The lines below parts (b) and (c) show the width of the region that was averaged to produce the profiles shown in Fig. 4.

spherical divergence is not significant. The numerical scaling between our experiment and the SNR is 1 ns to 1 year, 100 $\mathrm{km} / \mathrm{s}$ to $10^{4} \mathrm{~km} / \mathrm{s}$, and $100 \mu \mathrm{m}$ to 0.03 light years.

\section{EXPERIMENTAL METHODS}

We performed these experiments using the Nova laser. ${ }^{2}$ We used eight of the ten laser beams to inject about $20 \mathrm{~kJ}$ of laser energy, at a wavelength of $0.35 \mu \mathrm{m}$ and with constantintensity pulses of 1-ns duration, into the ends of a cylindrical, gold cavity that was $1.6 \mathrm{~mm}$ in diameter and $3 \mathrm{~mm}$ in length. This heated the cavity to about $220 \mathrm{eV}$. On the side of the cylinder, at its midplane, we mounted the experimental package shown above in Fig. 1. The soft $\mathrm{x}$ rays in the cavity deposit about $1 \mathrm{~kJ}$ of $\mathrm{x}$-ray energy into the plastic plug, launching a shock wave into it with an ablation pressure of about $50 \mathrm{Mbar}$. Two plug densities, 1.22 and $1.54 \mathrm{~g} / \mathrm{cc}$, were used. These were produced by varying the atomic concentration of $\mathrm{Br}$ in the $\mathrm{CH}$ from $2 \%$ to $6 \%$. The plug thickness was measured in each case, and ranged from 196 to $207 \mu \mathrm{m}$. The shock wave was observed (optically) to break out of a $205-\mu \mathrm{m}$ thick, $1.22 \mathrm{~g} / \mathrm{cc}$ plug at $2.9 \pm 0.1 \mathrm{~ns}$, and out of a $205-\mu \mathrm{m}$ thick, $1.54 \mathrm{~g} / \mathrm{cc}$ plug at $3.3 \pm 0.1 \mathrm{~ns}$.

After the ejecta from the plug crossed the gap of nominally $150 \mu \mathrm{m}$ (142 to $157 \mu \mathrm{m}$ for the data discussed here), it drove a shock into the $\mathrm{SiO}_{2}$ aerogel foam of density $40 \mathrm{mg} /$ cc. We measured the shock, the density profile, and the other features using x-ray radiography. To accomplish this, we irradiated a metal plate, of $\mathrm{Fe}$ or $\mathrm{Sc}$ for the data discussed here, with two of the Nova laser beams in succession. This produced $K_{\alpha}$ x rays at 6.7 or $4.3 \mathrm{keV}$, respectively. We used a pinhole camera, with an array of 16 pinholes of $10 \mu \mathrm{m}$ diameter, to view the backlit target at $8 \times$ magnification. Each pinhole produced an image on a microchannel-plate framing camera. ${ }^{32}$ A given group of four images was separated by 100 ps. The separation of the groups was measured, and was typically $1 \mathrm{~ns}$. The photons from the phosphor on the back of the framing camera were recorded on film, along with a calibration feature that allowed reconstruction of the x-ray intensity from the film density, which was digitized.

The analysis of the backlit $\mathrm{x}$-ray images proceeded as follows. We imaged the x-ray source, without any intervening material, in an arrangement identical to that used to obtain the hydrodynamic data. We then fit the shape of this source to a mathematical function that we used for data reduction. After trying a number of possible descriptions of the spot shape, we settled on a generalized, elliptical superGaussian profile with an underlying intensity ramp. The hydrodynamic data typically included at least one region of known, constant opacity, such as the uncompressed foam or the plastic base. (It proved unnecessary to account for the slight deviation from flatness produced by viewing the central $400 \mu \mathrm{m}$ of a $700-\mu \mathrm{m}$ diameter cylinder.) In order to determine the location of the backlighter spot, we used a least-squares method to minimize the deviation of the known region from a flat profile. In cases for which only the central portion of the backlighter spot was important, this proved sufficient.

In cases for which a large fraction of the backlighter spot was used, some further manual iteration was needed. An example of such a case is shown in Fig. $3{ }^{33}$ Shields produce the lateral limits of the images; the edge of the gated area on the microchannel plate produces the lower limit. In the raw data (a), one can make out the elliptical shape of the backlighter spot, with bright signal from lower left to upper right. The plastic base and the uncompressed foam each have a nearly constant optical depth. There is a range of backlighter 


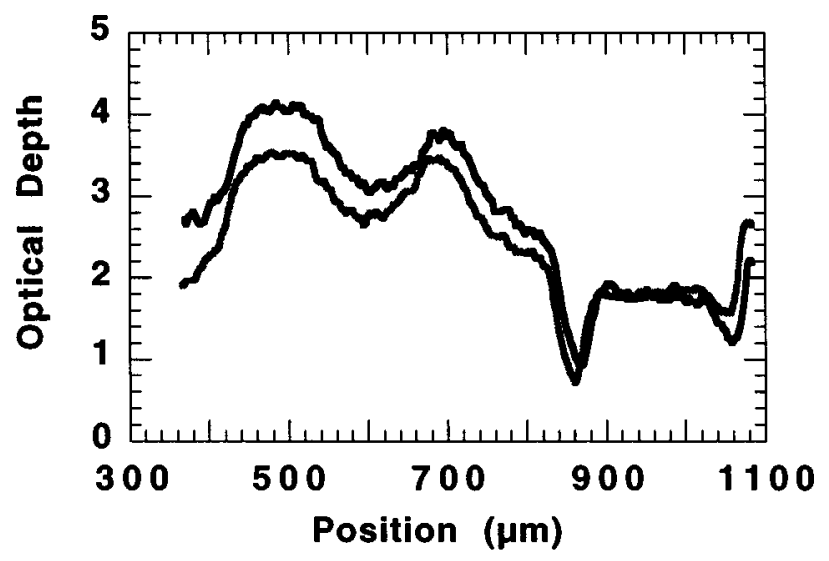

FIG. 4. Profiles of optical depth corresponding to the optical depth images in Fig. 3.

spot positions that produces this result. Parts (b) and (c) in Fig. 3 show the limits of this range, and thus indicate the uncertainty in the data reduction. One sees that the lateral uniformity is good in both cases, and that much of the vertical structure appears quite similar. However, the optical depth of the uppermost region in the target, which is seen through the edge of the backlighter spot, is significantly uncertain.

\section{EXPERIMENTAL RESULTS}

Figure 4 shows the vertical profiles of optical depth corresponding to the optical depth images in Fig. 3. One can see the slight differences in the profile of the plastic base, which extends from just below $900 \mu \mathrm{m}$ to above $1000 \mu \mathrm{m}$. The optical depth of this base should be flat, so the two cases shown correspond to limits on either side of the optimum. One sees that the profile of the shocked structure is very well measured in this case. The absolute optical depth of the plug is more uncertain than that of the shocked structure; a consequence of being further from the region used to position the backlighter spot. In addition, we note that while the pinhole resolution may be $10 \mu \mathrm{m}$, the resolution obtained here is approximately $30 \mu \mathrm{m}$, due to viewing these structures across the $700 \mu \mathrm{m}$ flat surfaces at some small angle.

One can see in Fig. 3 that the shocks appear quite planar over the observed $400-\mu \mathrm{m}$ width. We made additional measurements without shields, and in the orthogonal direction as well, to further assess the planarity and edge effects. As one would expect, we did observe curvature on the outer edges of the shock. The flat, central region pulls ahead of the edges. These results were also consistent with $2 \mathrm{D}$ simulations. We concluded that the effective optical depth of the observed plug material, along the diagnostic line of sight, is between 350 and $700 \mu \mathrm{m}$.

One can use this result to infer the density of the plug and of the ejecta peak from these data. The calibration reference is that $700 \mu \mathrm{m}$ of $\mathrm{C}_{50} \mathrm{H}_{48} \mathrm{Br}_{2}$, at $1 \mathrm{~g} / \mathrm{cc}$, has an optical depth of 8.1. This implies that the density at the maximum of the plug material in Fig. 4 is $0.72 \pm 0.28 \mathrm{~g} / \mathrm{cc}$. The corresponding density of the ejecta peak is $0.68 \pm 0.25 \mathrm{~g} / \mathrm{cc}$. These

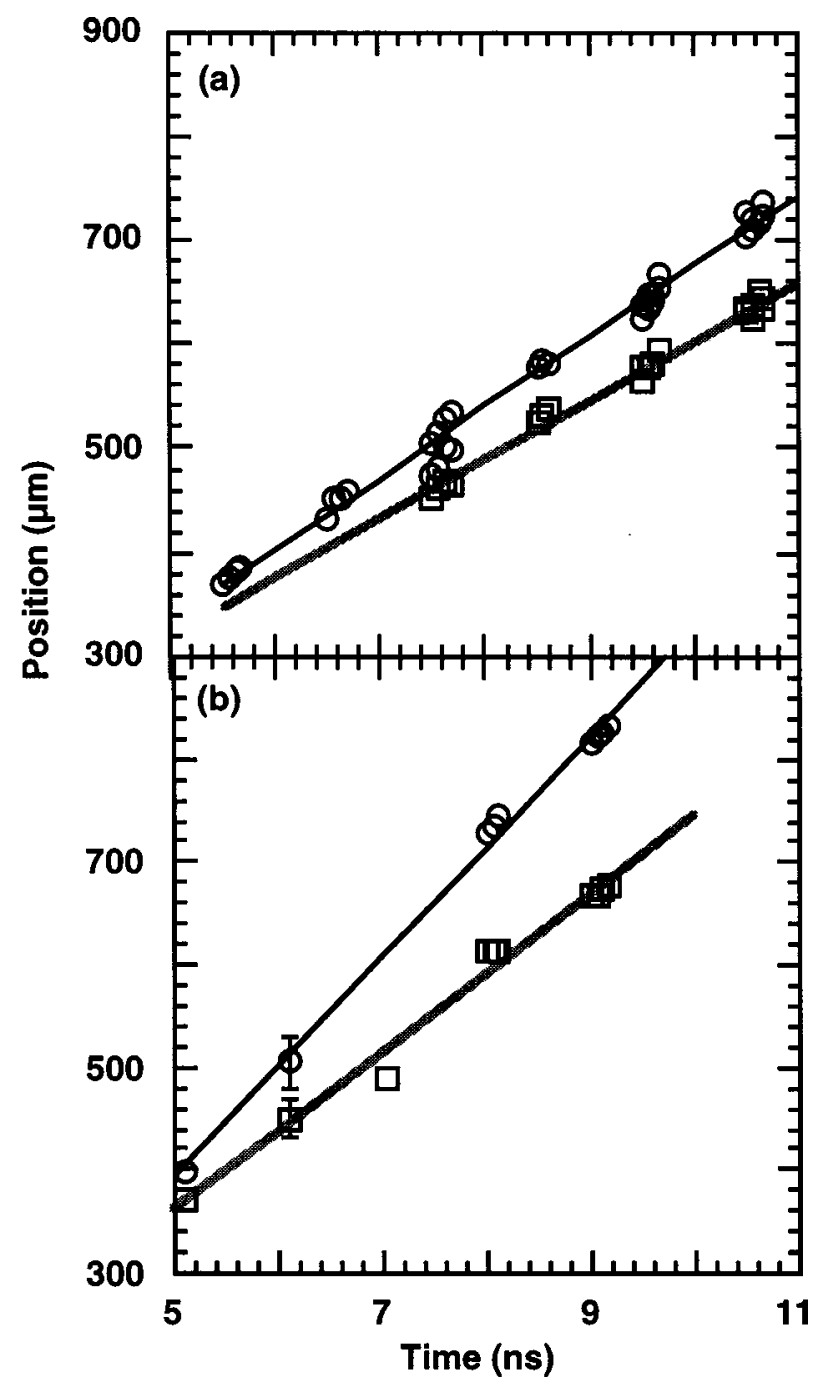

FIG. 5. The positions of the forward shock (circles) and of the ejecta peak (squares) are shown vs time for experiments with (a) the $1.54 \mathrm{~g} / \mathrm{cc}$ plug and (b) the $1.22 \mathrm{~g} / \mathrm{cc}$ plug. The error bars are smaller than the symbols except in the two cases shown.

results, and the profiles shown in Fig. 4, can be compared with those produced by simulations. The optical depth of the uncompressed foam is 0.580 , from which the density of the shocked foam is found, by the same calculation, to be 0.15 to $0.36 \mathrm{~g} / \mathrm{cc}$. A better evaluation of the shocked-foam density, from the shock properties, is discussed next. It gives a density of $0.16 \pm 0.04 \mathrm{~g} / \mathrm{cc}$.

Our data allows us to determine several properties of the shocked foam by a standard hydrodynamic analysis. We obtained a sequence of images like those shown in Fig. 3, at different times. This allowed us to plot the position of the forward shock and of the ejecta peak vs time. These data, with least-squares fits to the velocity, are shown in Fig. 5. (In a previous publication, ${ }^{13}$ these data have been compared to simulations.) We note that, although the data would allow some slowing, deceleration of these two features is not evident. The velocity of the ejecta peak can be used as a measure of the "particle velocity" - the fluid velocity behind the shock front. Simulations show that the actual velocity behind the shock front, at early times, is less than $2 \%$ larger than the 


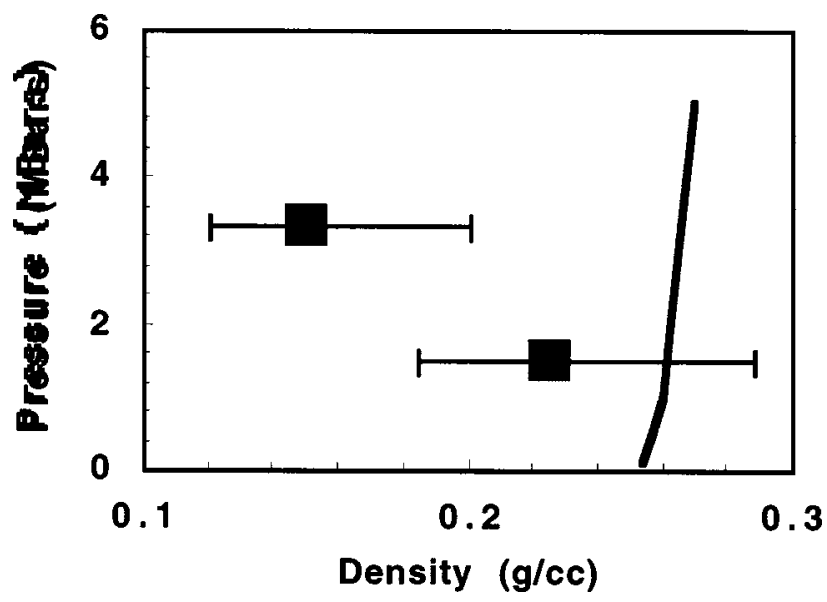

FIG. 6. pressure vs density of shocked foam. The points show the parameters of shocked, $40 \mathrm{mg} / \mathrm{cc}$, silicate aerogel foam determined from measurements as described in the text. The vertical error bars lie within the points. The curve shows results from the standard SESAME library equation of state table, for $\mathrm{SiO}_{2}$.

ejecta-peak velocity. We used 1.00 and 1.03 times the ejectapeak velocity to place limits on the results. Then, knowing the shock velocity, the particle velocity, and the initial density, we can determine the shocked density, shocked pressure, and effective adiabatic coefficient, $\gamma$, as usual. The results of this analysis are shown in Fig. 6. The corresponding values of $\gamma$ are $1.74 \pm 0.26$ for a $107-\mathrm{km} / \mathrm{s}$ shock and 1.44 \pm 0.12 for a $68-\mathrm{km} / \mathrm{s}$ shock. (The implied compression can be larger than fourfold, because energy is required during compression to atomize and ionize the radiating foam material.) As the curve shows, the SESAME tables, which are scaled from the properties of solid density $\mathrm{SiO}_{2}$, would predict significantly more compression than we observe.

The data (e.g., Fig. 3) also show the motion of the residual plug mass through the system, for the experiments using a plug density of $1.22 \mathrm{~g} / \mathrm{cc}$. Based on profiles like those shown in Fig. 4, we determined the points at which the optical depth was $75 \%$ of the maximum in this feature. These are shown in Fig. 7. The scatter in the points clustered near the same location, which are obtained from a sequence of frames spaced by $100 \mathrm{ps}$, indicates the reproducibility of the analysis. Figure 7 also shows four curves. The dashed lines are least-squares linear fits to the two sets of data. The outer edge of the plug moves at $88 \pm 3 \mathrm{~km} / \mathrm{s}$, while the inner edge moves at $76 \pm 2 \mathrm{~km} / \mathrm{s}$. One can see that there is no evidence here of acceleration or deceleration of the plug during the interval shown. However, the other two lines, discussed next, have the implication that the plug has been strongly accelerated after the action of the initial shock.

The solid and dot-dash lines provide definitive evidence of postshock acceleration of the plug mass. The solid line shows the position where the center of mass of the plug would be, assuming that the shock velocity in the plug was constant and that this mass moved at constant velocity from the moment of the observed shock breakout. Specifically, the shock breakout time (2.9 ns) was used to infer the material velocity $(52 \mathrm{~km} / \mathrm{s}, 3 / 4$ of the shock velocity) of the center of mass of the plug (at $175 \mu \mathrm{m}$ at shock breakout). This line is

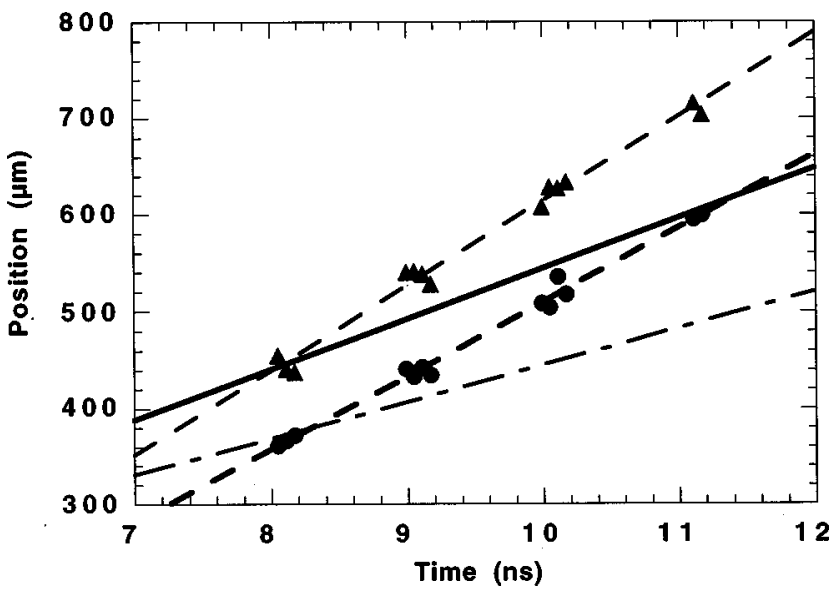

FIG. 7. The variation with time of the position of the outer (triangles) and inner (circles) $75 \%$ of maximum plug density is shown, for the case of a $1.22 \mathrm{~g} / \mathrm{cc}$ plug. The lines are upper limits on the center-of-mass position based on shock breakout (solid) and center-of-mass velocity at breakout from a simulation (dot-dash).

an absolute upper limit on the plug location, in the absence of postshock acceleration.

The lower, dot-dash line shows another overestimate of the distance the plug will have moved, but a more realistic one, requiring input from simulations in addition to experimental data. The actual velocity of the plug material is smaller than the solid line indicates because the shock slows down before it breaks out, and because the material we detect as "the plug" does not include the high-velocity matter that becomes the flowing and stagnated ejecta. We can account for the first effect by using a simulation that obtains the correct shock breakout time from a radiation drive pulse to determine the center-of-mass fluid velocity at that time (38 $\mathrm{km} / \mathrm{s}$ ). Motion with this velocity is shown by the dot-dash line in Fig. 7. It is quite clear from Fig. 7 that there must have been a strong acceleration before $8 \mathrm{~ns}$. During the 8 to $12 \mathrm{~ns}$ interval, the velocity of the plug is much faster than either of these overestimates of the velocity due to the initial shock. In conclusion, we have here strong, direct evidence that the plug was accelerated after shock breakout (2.9 ns) but before $8 \mathrm{~ns}$.

In the other experimental case, the plug whose initial density was $1.54 \mathrm{~g} / \mathrm{cc}$ moved much more slowly. In consequence, it did not move far enough to see clearly until quite late in the experiment. We did obtain one good measurement of its position. This result is shown in Fig. 8. The solid line in Fig. 8 shows the upper-limit center of mass trajectory for the denser plug, in this case based on a shock breakout time of $3.3 \mathrm{~ns}$, giving an average material velocity of $45 \mathrm{~km} / \mathrm{s}$. The dot-dash line again shows the estimate based on simulations, which gave a center-of-mass velocity, at $4 \mathrm{~ns}$, of 29 $\mathrm{km} / \mathrm{s}$. Here again, the evidence is clear that the plug mass was further accelerated after the initial shock.

\section{ANALYSIS AND DISCUSSION}

The immediate question raised by these data is the origin of the second impulse that further accelerates the plug mass. We attribute this to the stagnation of plasma at the center of 


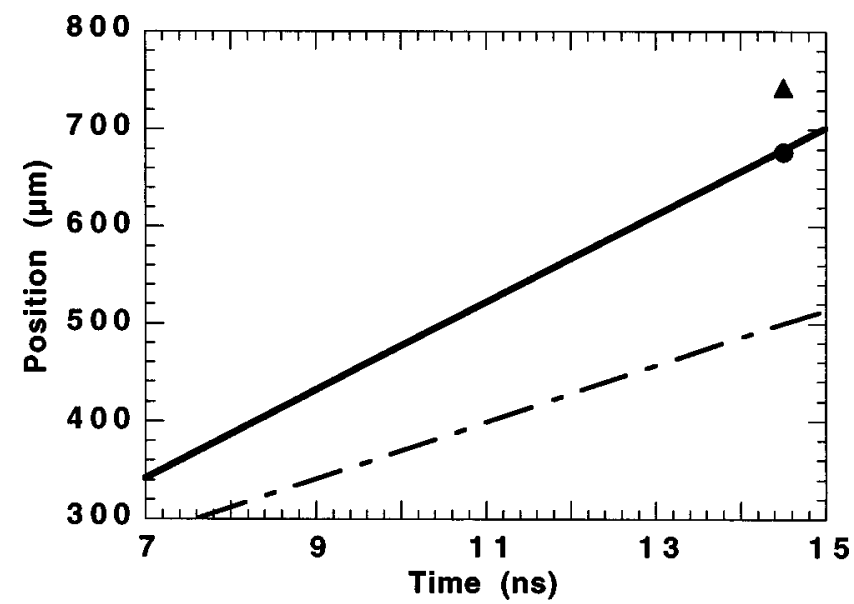

FIG. 8. The position of the outer (triangle) and inner (circle) $75 \%$ of maximum plug density is shown, for the case of a $1.54 \mathrm{~g} / \mathrm{cc}$ plug. The lines are upper limits on the center-of-mass position based on shock breakout (solid) and center-of-mass velocity at breakout from a simulation (dot-dash).

the hohlraum. The hohlraum is $800 \mu \mathrm{m}$ in radius, so that matter moving a few hundred $\mathrm{km} / \mathrm{s}$ reaches the origin in very few ns. The matter at the center of the hohlraum will be dominated by dense, gold plasma, and the resulting pressure pulse can very plausibly launch a shock up the plastic density ramp that extends from the plug to the hohlraum axis. Based on the data and simulations, this second shock must overtake, and coalesce with, the first one after the first shock breaks out of the plug but before the observed forward and reverse shocks are established in the foam.

Our approach to this issue is an empirical one. We are seeking to define a pressure pulse that, when added to the radiation pulse, will reproduce the observations. Because the data regarding the plug motion, the ejecta peak, and the forward shock are quite extensive, we expect to find that only a narrow range of possible pulses will produce this result. Once we have thus established the initial conditions that reproduce the data, further work will proceed in two directions. First, modeling of the hohlraum environment can attempt to understand how this pressure pulse is produced. This may prove difficult using the current, $2 \mathrm{D}$ codes to approximate this 3D phenomenon. Second, the initial conditions we develop can be used to initiate multidimensional modeling of hydrodynamic instabilities in this system.

Our progress to date at defining such a pressure pulse is shown in Fig. 9. We are using the HYADES radiation hydrodynamics code for this work. This is a 1D, Lagrangian code, run with graybody radiation transport. Because a much more sophisticated model is needed to handle the radiation ablation correctly for the case of a $1.22 \mathrm{~g} / \mathrm{cc}$ plug, ${ }^{13}$ we adjusted the radiation input to achieve the correct initial shock breakout time. We then added a pressure pulse at the left boundary. In the case shown, this pressure pulse increased abruptly to $38 \mathrm{Mbar}$ at $1.5 \mathrm{~ns}$, decreased linearly to $25 \mathrm{Mbar}$ in $5 \mathrm{~ns}$, then decreased linearly to zero over $1 \mathrm{~ns}$. The foam was treated as an ideal gas with an adiabatic coefficient of $5 / 3$, because of the discrepancy with the SESAME table discussed above. As Fig. 9 shows, the added pressure pulse does create an accumulation of plug mass similar to that seen

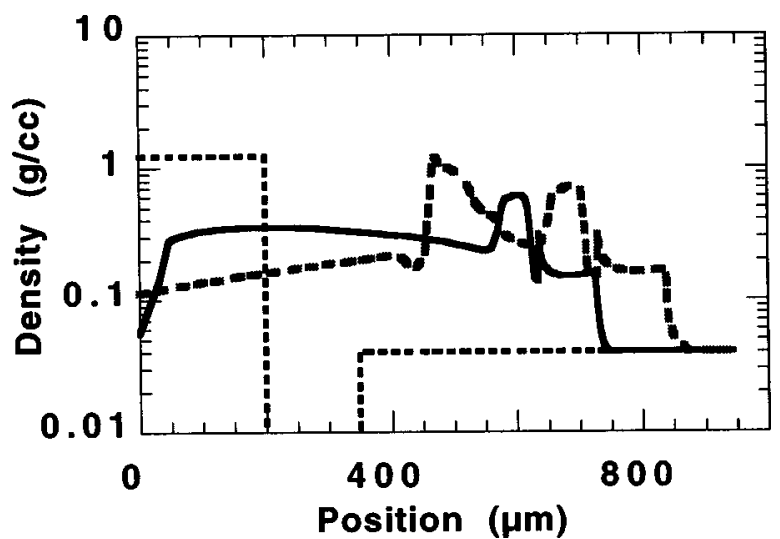

FIG. 9. Modeling of the density profile. The short-dash curve shows the initial density profile. The solid curve shows the profile obtained using only a radiation source. The dashed, gray curve shows the profile obtained by adding the additional pressure pulse described in the text.

in the data. When making the comparison, note that the spatial resolution in the data is only about $30 \mu \mathrm{m}$, as discussed above.

\section{CONCLUSION}

We have created an experimental system that is well scaled from the laboratory to the environment of SN1987A. We have studied the behavior of the laboratory system using $\mathrm{x}$-ray radiography. The matter is accelerated by an $\mathrm{x}$-ray radiation pulse and by an additional impulse, revealed by the data, that we attribute to hohlraum stagnation. Our goal is to achieve a sufficiently complete characterization of this system so that we can provide an accurate and complete set of initial conditions that will allow any hydrodynamic code to model its behavior. We are close to achieving this goal. It will make possible the use of multidimensional astrophysical codes to model the evolution of hydrodynamic instabilities, for comparison with data.

\section{ACKNOWLEDGMENTS}

The authors acknowledge the efforts of the technical staff of the Nova laser, without whom this work would not have been possible. In addition, at Michigan, the contributions of Korbie Dannenberg, Harry Reisig, Kristy Brock, and Nicolai Maslov are acknowledged. This work was supported by the Department of Energy both directly and through the Lawrence Livermore National Laboratory, and by the University of Michigan.

${ }^{1}$ J. D. Lindl, Inertial Confinement Fusion (Springer, New York, 1998).

${ }^{2}$ E. M. Campbell, J. T. Hunt, E. S. Bliss, D. R. Speck, and R. P. Drake, Rev. Sci. Instrum. 57, 2101 (1986).

${ }^{3}$ J. M. Soures, R. L. McCrory, C. P. Verdon, A. Babushkin, R. E. Bahr, T. R. Boehly, R. Boni, D. K. Bradley, D. L. Brown, R. S. Craxton, J. A. Delettrez, W. R. Donaldson, R. Epstein, P. A. Jaanimagi, S. D. Jacobs, K. Kearney, R. L. Keck, J. H. Kelly, T. J. Kessler, R. L. Kremens, J. P. Knauer, S. A. Kupman, S. A. Letzring, D. J. Lonobile, S. J. Loucks, L. D. Lund, F. J. Marshall, P. W. McKenty, D. D. Meyerhofer, S. F. B. Morse, A. Okishev, S. Papernov, G. Pien, W. Seka, R. W. Short, M. J. Shoup, M. D. Skeldon, S. Skupsky, A. W. Schmid, D. J. Smith, S. Swales, M. Wittman, and B. Yaakobi, Phys. Plasmas 3, 2108 (1996).

${ }^{4}$ R. P. Drake, J. Geophys. Res. 104, 14 (1999). 
${ }^{5}$ J. Grun, J. Stamper, C. Manka, J. Resnick, R. Burris, J. Crawford, and B. H. Ripin, Phys. Rev. Lett. 66, 2738 (1991).

${ }^{6}$ J. Kane, D. Arnett, B. A. Remington, S. G. Glendinning, J. Castor, R. Wallace, A. Rubenchik, and B. A. Fryxell, Astrophys. J. 478, L75 (1997).

${ }^{7}$ J. Kane, D. Arnett, B. A. Remington, S. G. Glendinning, G. Bazan, R. P. Drake, B. A. Fryxell, R. Teyssier, and K. Moore, Phys. Plasmas 6, 2065 (1999).

${ }^{8}$ A. M. Khokhlov, E. S. Oran, and J. C. Wheeler, Combust. Flame 105, 28 (1996).

${ }^{9}$ A. M. Khokhlov, E. S. Oran, and G. O. Thomas, Combust. Flame 117, 323 (1999).

${ }^{10}$ B. A. Remington, R. P. Drake, D. Arnett, and H. Takabe, Science 284, 1488 (1999).

${ }^{11}$ B. A. Remington, R. P. Drake, D. Arnett, and H. Takabe, Phys. Plasmas 7, 1641 (2000); also Bull. Am. Phys. Soc. 44, 17 (1999).

${ }^{12}$ R. P. Drake, J. J. Carroll, K. Estabrook, S. G. Glendinning, B. A. Remington, and R. McCray, Astrophys. J. Lett. 500, L157 (1998).

${ }^{13}$ R. P. Drake, S. G. Glendinning, K. Estabrook, B. A. Remington, R. McCray, R. Wallace, L. J. Suter, T. B. Smith, R. London, and E. Liang, Phys. Rev. Lett. 81, 2068 (1998).

${ }^{14}$ R. A. Chevalier, Astrophys. J. 258, 790 (1982).

${ }^{15}$ J. P. Ostriker and C. F. McKee, Rev. Mod. Phys. 60, 1 (1988).

${ }^{16}$ R. A. Chevalier, J. M. Blondin, and R. T. Emmering, Astrophys. J. 392, 118 (1992).

${ }^{17}$ J. I. Reed, J. J. Hester, A. C. Fabian, and P. F. Winkler, Astrophys. J. 440, 706 (1995).
${ }^{18}$ D. A. Allen and M. G. Burton, Nature (London) 363, 54 (1993).

${ }^{19}$ M.-M. MacLow, Nature (London) 377, 287 (1995).

${ }^{20}$ J. M. Stone, J. Xu, and L. G. Mundy, Nature (London) 377, 315 (1995).

${ }^{21}$ R. McCray and D. N. C. Lin, Nature (London) 369, 378 (1994).

${ }^{22}$ D. Luo and R. McCray, Astrophys. J. 379, 659 (1991).

${ }^{23}$ L. Wang and P. A. Mazzali, Nature (London) 355, 58 (1992).

${ }^{24}$ J. M. Blondin and P. Lundqvist, Astrophys. J. 405, 337 (1993).

${ }^{25}$ R. Chevalier and Dwarkadas, Astrophys. J. 452, L45 (1995).

${ }^{26}$ B. M. Gaensler, R. N. Manchester, L. Staveley-Smith, A. K. Tzioumis, J. E. Reynolds, and M. J. Kesteven, Astrophys. J. 479, 845 (1997).

${ }^{27}$ G. Sonneborn, C. S. J. Pun, R. A. Kimble, T. R. Gull, P. Lundqvist, R. McCray, P. Plait, A. Boggess, C. W. Bowers, A. C. Danks, J. Grady, S. R. Heap, S. Kraemer, D. Lindler, J. Loiacono, S. P. Maran, W. W. Moos, and B. E. Woodgate, Astrophys. J. Lett. 492, L139 (1998).

${ }^{28}$ T. Suzuki, T. Shigeyama, and K. Nomoto, Astron. Astrophys. 274, 883 (1993).

${ }^{29}$ K. J. Borkowski, J. M. Blondin, and R. McCray, Astrophys. J. 477, 281 (1997).

${ }^{30}$ K. J. Borkowski, J. M. Blondin, and R. McCray, Astrophys. J. 476, L31 (1997).

${ }^{31}$ D. D. Ryutov, R. P. Drake, J. Kane, E. Liang, B. A. Remington, and M. Wood-Vasey, Astrophys. J. 518, 821 (1999).

${ }^{32}$ K. S. Budil, T. S. Perry, P. M. Bell, J. D. Hares, P. L. Miller, T. A. Peyser, R. Wallace, H. Louis, and D. E. Smith, Rev. Sci. Instrum. 67, 485 (1996).

${ }^{33}$ For reference, this is strip 2, frame 3 from experiment 28020511 at $9.11 \mathrm{~ns}$. 